\section{Showcasing a smile}

Align Technology has announced the launch of SmileView, an online tool, designed to help prospective Invisalign patients visualise a new, straighter smile before they opt for Invisalign treatment. Align's smile modelling tool is being showcased at the British Dental Conference and Dentistry Show.

SmileView tool is a computer-generated simulation of how a person's smile may look after teeth straightening. Within 60 seconds of taking a smiling selfie using the SmileView online tool on their smartphone or tablet, prospective patients can see what their new smile and straighter teeth may look like, with their own facial features.

Using a highly sophisticated machine learning algorithm, the automated system leverages a database of tooth movements from over 6 million Invisalign cases to provide a simulation of the person's dentition and projected outcome.

Align's new SmileView visualisation tool is designed to drive awareness and demand for teeth straightening using Invisalign treatment by engaging consumers and allowing them to see a simulation of what their new smile could look like. For those who request a consultation with an Invisalign doctor as the next step, SmileView tool will direct the users to the nearest doctors in their area via Find a Doctor tool or alternatively, via the Company's or Align's Smile Concierge team that can help consumers find an experienced doctor in their area and schedule an appointment.

For more information about the Invisalign system and to experience SmileView tool, visit www.invisalign.co.uk

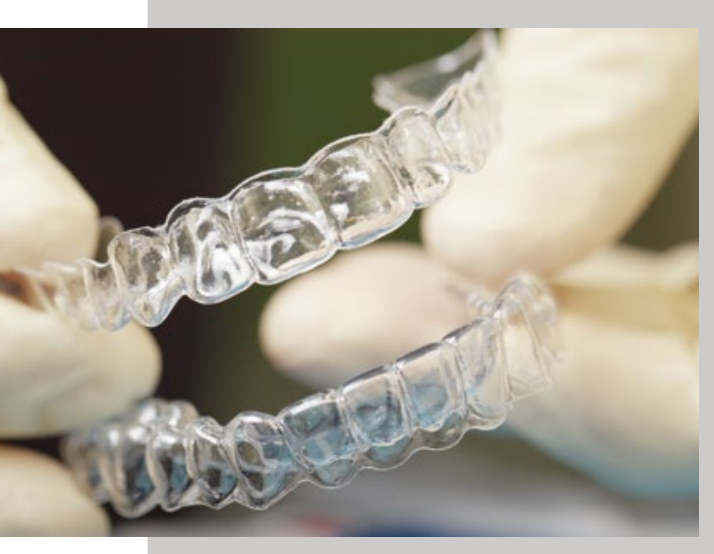

\title{
Time for a transformation!
}

Whether you need equipment, uniforms, imaging technology, or a complete refurbishment, call RPA Dental.

The team has a proud history of supporting the dental community in bringing all kinds of projects, big and small, to successful completion.

This year, RPA Dental will be returning to the British Dental Conference and Dentistry
Show at NEC Birmingham. Delegates can see its stunning portfolio and, as RPA Dental is the distributor for Castellini, see the newly re-launched Castellini Skema 8 dental unit, the complete treatment centre for every forward-thinking dentist.

Make a visit stand N50 an absolute must!

Visit www.rpadental.net for further information.

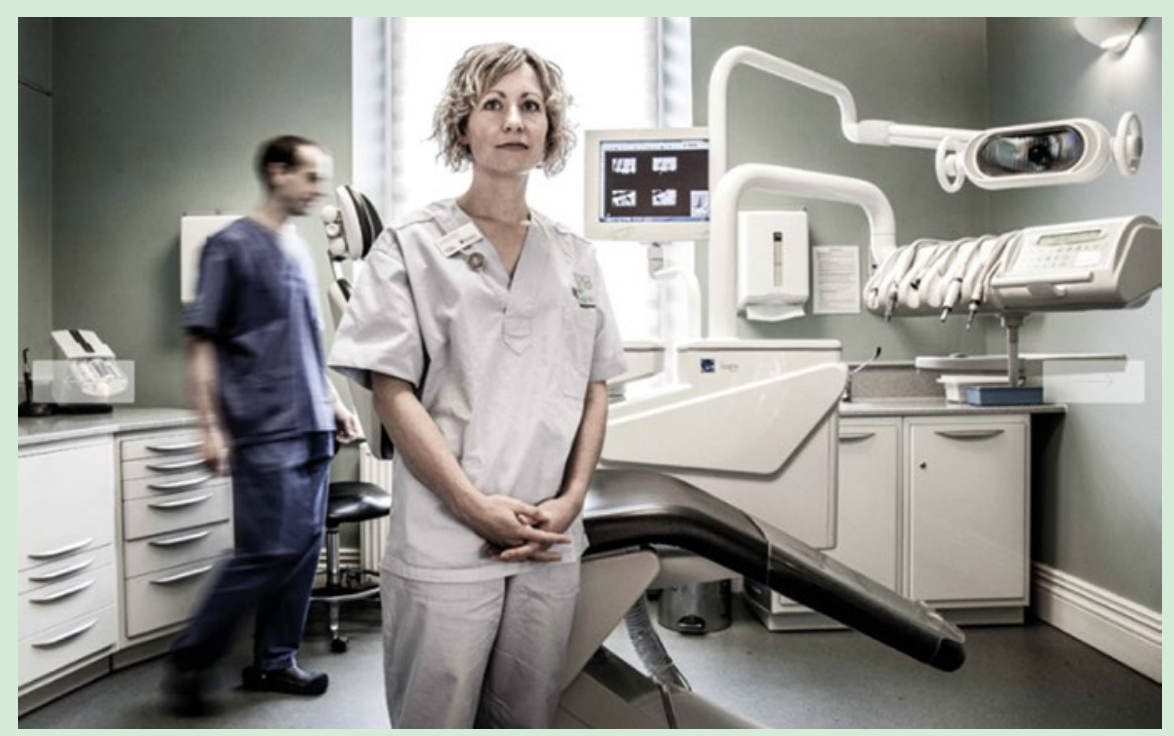

\section{The easy way to clean}

Being clinically proven to reduce gingival disease, it's no wonder the Wisdom Clean Between Interdental Brushes are the UK's No.1 selling rubber interdental brushes.

But that's not all Wisdom Toothbrushes has to offer - visit the team at the British Dental Conference and Dentistry Show 2019 to find out more.

Discover the Wisdom Pro-flex Wire Interdental Brushes, featuring a unique flexible hinge and curved profile to easily reach all areas of the mouth.

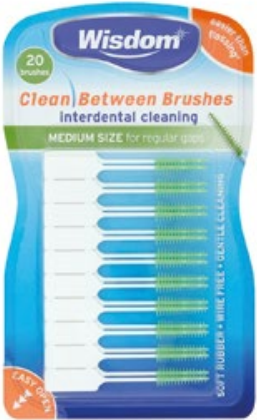

Or, perhaps your patients prefer the Wisdom Clean Between Easy Slide Tensioning Flossers with the unique patented Waveform Tension Control System, which holds the silk-like tape taunt, thereby ensuring effortless and comfortable interdental cleaning.

For this and much more, drop by stand P75.

To find out more, visit www. wisdomtoothbrushes.com or call 01440 714800 . 